\title{
Search for new physics in bosonic final states at the LHC
}

\section{Andreas Hinzmann*}

On behalf of the ATLAS, CMS and LHCb Collaborations

University of Zurich

E-mail: hinzmannecern.ch

\begin{abstract}
A summary of recent searches by the ATLAS, CMS and LHCb experiments at the LHC for new physics signatures that involve the production of bosons is given. The results are based on the first data collected at the LHC in 2015 at a center of mass energy of $13 \mathrm{TeV}$, corresponding to an integrated luminosity of 2.2-3.2/fb. Statistical combinations with previous data collected at $8 \mathrm{TeV}$ are also presented. Particular focus is given to two hints of resonances in di-boson final states at $750 \mathrm{GeV}$ and $2 \mathrm{TeV}$.
\end{abstract}

Fourth Annual Large Hadron Collider Physics

13-18 June 2016

Lund, Sweden

${ }^{*}$ Speaker. 


\section{Introduction}

After the discovery of the Higgs boson, the primary goal of the ATLAS [1], CMS [2] and LHCb [3] experiments is the discovery of new physics beyond the standard model (BSM). In 2015, the LHC produced proton-proton collisions at a center of mass energy of $13 \mathrm{TeV}$ for the very first time, allowing to explore new physics signatures at yet higher energies than reachable in the 2012 run of the LHC at a center of mass energy of $8 \mathrm{TeV}$. Sensitivity to new physics signatures with invariant masses above $\sim 1 \mathrm{TeV}$ is already better than in 2012 and thus territory for discoveries, taking into account that the amount of data collected in 2015 corresponding to an the integrated luminosity of 2.3-3.2/fb for ATLAS and CMS is about 1/6 of that collected in 2012.

Due to their potential relation to the electroweak symmetry breaking mechanism, new physics signatures that involve the production of bosons are of particular interest. Multiple models that offer a solution to the hierarchy problem, including super symmetry, extra dimensions and compositeness, predict new resonances decaying to combinations of $\gamma, \mathrm{W}, \mathrm{Z}$ or Higgs bosons. Theories with hidden-sector particles that may resolve our current lack of evidence for a dark matter particle candidate, predict new low mass bosons. Therefore, ATLAS, CMS and LHCb have a wide program of searches for signatures that involve the production of bosons that is summarized in this contribution.

Recent excitement in di-boson searches came from a hint of an excess in the $\gamma \gamma$ invariant mass spectrum at $750 \mathrm{GeV}$ in the 2015 data, seen by ATLAS with a local significance of $3.9 \sigma$ and by CMS with $3.4 \sigma$. Also a hint of an excess at $\sim 2 \mathrm{TeV}$, seen by ATLAS in 2012 data with a local significance of $3.4 \sigma$ in the $\mathrm{WZ}$ invariant mass spectrum and by CMS with $2.2 \sigma$ in the $\mathrm{WH}$ invariant mass spectrum, raised high interest in the analysis of the 2015 data, presented here.

\begin{tabular}{|c|c|}
\hline Signature & final state and references \\
\hline \multirow[t]{2}{*}{$\gamma \gamma$} & $\gamma \gamma(\operatorname{ATLAS~} 13 \mathrm{TeV})[4,5], \gamma \gamma(\mathrm{CMS} 13 \mathrm{TeV})[6,7]$ \\
\hline & $\gamma \gamma($ ATLAS $8 \mathrm{TeV})[8,9], \gamma \gamma(\mathrm{CMS} 8 \mathrm{TeV})[10,11]$ \\
\hline \multirow[t]{2}{*}{$\gamma Z$} & $\gamma \mathrm{Z}$ (ATLAS $13 \mathrm{TeV}$ ) [12], $\gamma \mathrm{qq}(\mathrm{CMS} 13 \mathrm{TeV})$ [13], \\
\hline & $\gamma 11(\mathrm{CMS} 8+13 \mathrm{TeV})$ [14] $\gamma 11$ (ATLAS $8 \mathrm{TeV})[15]$ \\
\hline WW/WZ/ZZ & $\begin{array}{l}\text { 11/lv/vv/qq+qq (ATLAS } 13 \mathrm{TeV})[16], 1 v / q q+q q(\mathrm{CMS} 13 \mathrm{TeV})[17,18] \\
11 / 1 v / v v / \mathrm{qq}+\mathrm{qq} / \mathrm{bb}(\mathrm{CMS} 8+13 \mathrm{TeV})[19]\end{array}$ \\
\hline $\mathrm{WH} / \mathrm{ZH}$ & $\begin{array}{l}\text { 11/lv/vv+bb (ATLAS } 13 \mathrm{TeV})[20], 11 / 1 v / v v+\mathrm{bb}(\mathrm{CMS} 13 \mathrm{TeV})[21] \\
11 / 1 v / v v / \mathrm{qq}+\mathrm{qq} / \mathrm{bb}(\mathrm{CMS} 8+13 \mathrm{TeV})[19]\end{array}$ \\
\hline $\mathrm{HH}$ & bbbb (ATLAS $13 \mathrm{TeV}$ ) [22] \\
\hline
\end{tabular}

Table 1: List of recent searches for resonant boson pair production with high invariant masses at ATLAS and CMS.

This contribution therefore focuses on $\gamma \gamma$ and $Z \gamma$ resonance searches at energies above $\sim 200$ $\mathrm{GeV}$ and resonant $\mathrm{W} / \mathrm{Z} / \mathrm{H}$ boson pair production at energies beyond $\sim 1 \mathrm{TeV}$. In this regime of invariant resonance masses, the two outgoing W/Z/H bosons obtain a large Lorentz boost that leads to a special signature in the detector. Since the two products from the W/Z/H boson decay are close-by in angle, they require special identification techniques explained further in Section 2. Table 1 summarizes the most recent searches for resonant boson pair production at the time of 
this conference that are detailed in this contribution. In addition, a recent search for a low mass new boson resonance [23] is discussed, illustrating a complementary approach to the search for new physics. The program of lower mass W/Z/H boson pair production searches is summarized in another contribution to these proceedings with focus on BSM Higgs searches.

\section{Boosted boson identification}

In more than $60 \%$ of the cases, W/Z/H bosons decay into a quark anti-quark pair, which makes the reconstruction of such decays an essential ingredient for searches involving these bosons. For highly boosted W/Z/H bosons the shower of hadrons originating from the quark anti-quark pair merges into a single large radius jet of particles. Such jets can be distinguished from background jets initiated from single quarks or gluons by means of jet substructure techniques. Both ATLAS and CMS use two distinct jet substructure properties to identify boosted W/Z/H bosons, shown in Fig. 1; firstly, the reconstructed mass of a jet, and secondly, an additional observable that quantifies how likely a jet is composed of two hard structures rather than just one.
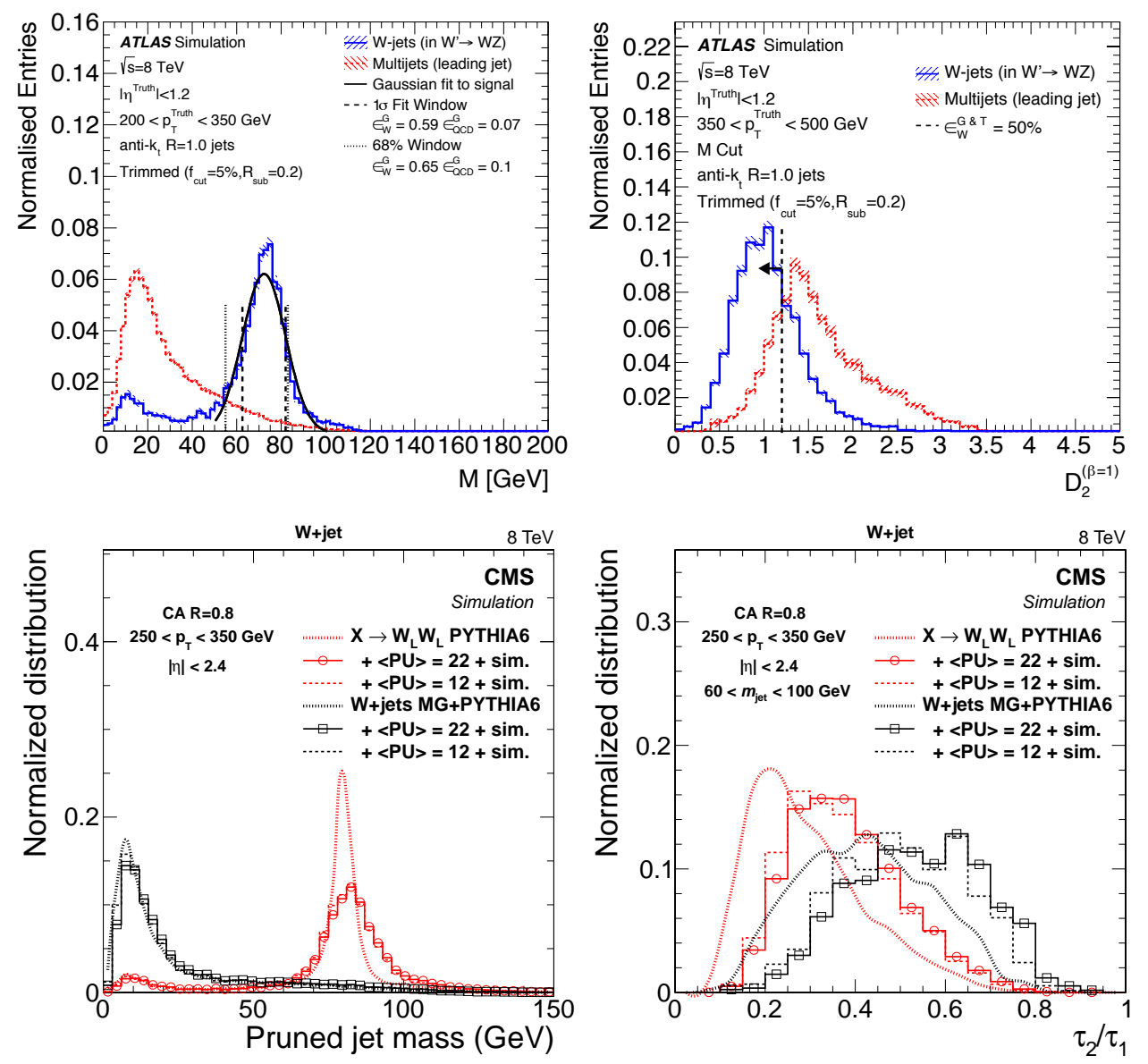

Figure 1: Boosted boson tagging observables used by (top) ATLAS [24] and (bottom) CMS [25].

In ATLAS, the jet mass is reconstructed using the trimming technique [24], that removes soft QCD radiation from the jet and thereby significantly reduces the jet mass of single quark or gluon 
jets, while maintaining the jet mass of boosted $\mathrm{W} / \mathrm{Z} / \mathrm{H}$ jets that contain two hard structures. CMS uses the pruning technique [25] to reconstruct the jet mass that similarly removes soft large-angle radiation from the jet, obtaining similar rejection power. To quantify the probability of a jet to be composed of two hard structures rather than just one, ATLAS relies on an energy correlation ratio $D_{2}$, while CMS relies on the N-subjettiness ratio $\tau_{2} / \tau_{1}$, again obtaining similar rejection power.

For the identification of $Z \rightarrow b \bar{b}$ and $H \rightarrow b \bar{b}$ decays, both ATLAS [26] and CMS [27] use special b-tagging techniques adapted to these final states, where either two small radius trackjets (ATLAS) or two subjets (CMS) inside the large radius jet are examined to determine whether they contain particles from a displaced B-meson decay.

\section{Di-photon resonances}

ATLAS has performed two complementary analyses to search for high mass resonances decaying to $\gamma \gamma$, shown in Fig. 2. One analysis is optimized to search for spin-0 resonances, while the other is optimized for spin-2 resonances. The analyses also rely on different background estimation strategies, serving as robust cross check for each other.
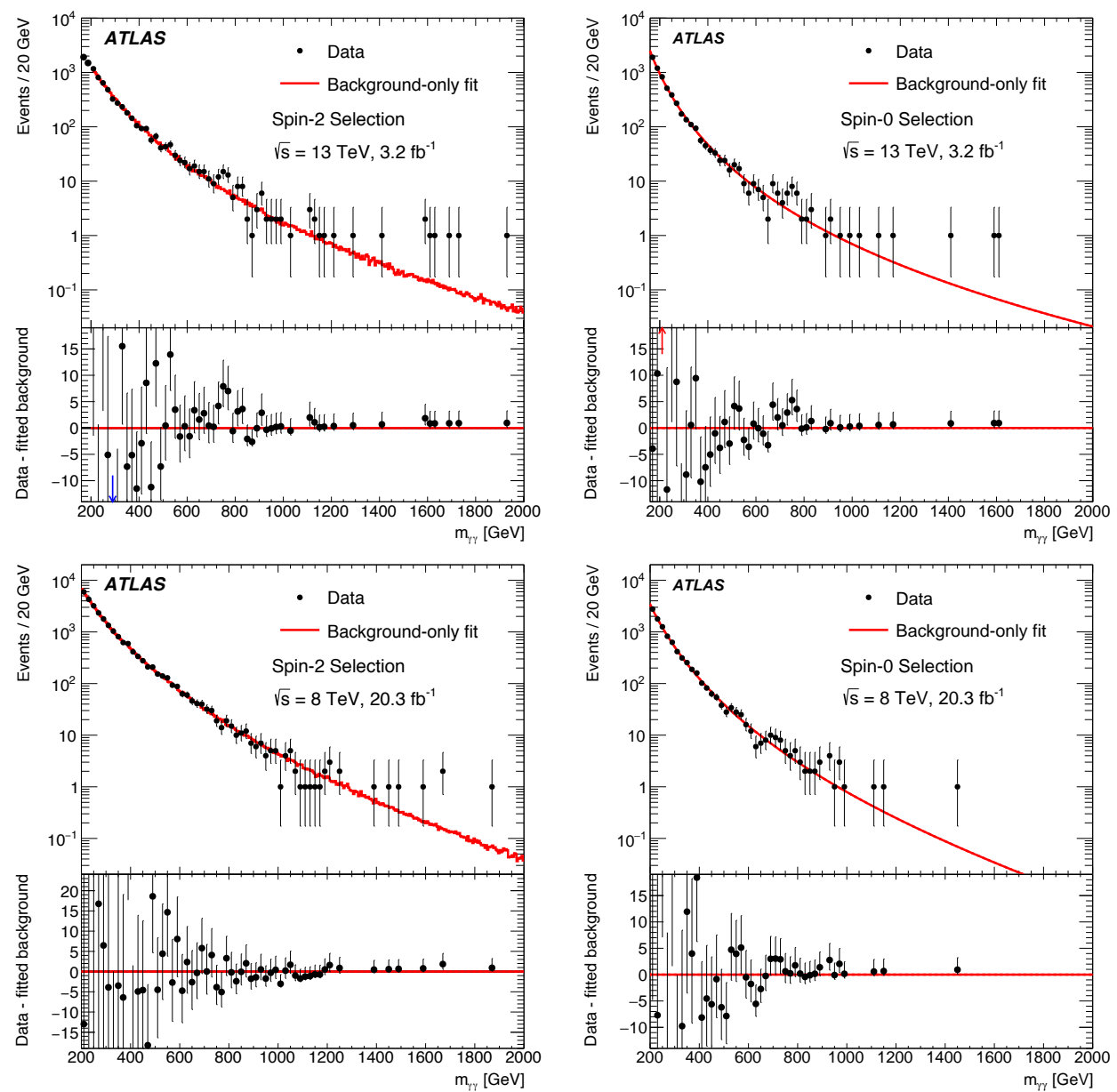

Figure 2: Di-photon invariant mass spectra of the ATLAS searches at $13 \mathrm{TeV}$ and $8 \mathrm{TeV}$ [5]. 
In the spin-0 analysis, events with two photons with $E_{T}>40(30) \mathrm{GeV}$ and $E_{T}>0.4(0.3) m_{\gamma \gamma}$ are selected. The latter cut is optimized for the decay kinematics of a spin- 0 resonance and enhances the sensitivity. The background is estimated from a signal + background fit, where the background is described by a smooth function: $f_{(k)}\left(x ; b, a_{k}\right)=N\left(1-x^{1 / 3}\right)^{b} x^{\sum_{j=0}^{k} a_{j}(\log x)^{j}}$ with $x=\frac{m_{\gamma \gamma}}{\sqrt{s}}, k=0$. This function models the sum of the main background component from standard model $\gamma \gamma$ production and also $\gamma+$ jets and QCD multijet production, where a jet is misidentified as a $\gamma$.

In the spin- 2 analysis, events with two photons with $E_{T}>55 \mathrm{GeV}$ are selected and no additional selection specific to the resonance kinematics is applied. The $\gamma \gamma$ background is estimated from a NLO theory prediction, while the $\gamma+$ jets and QCD multijets background are obtained from sidebands in $\gamma$ isolation.

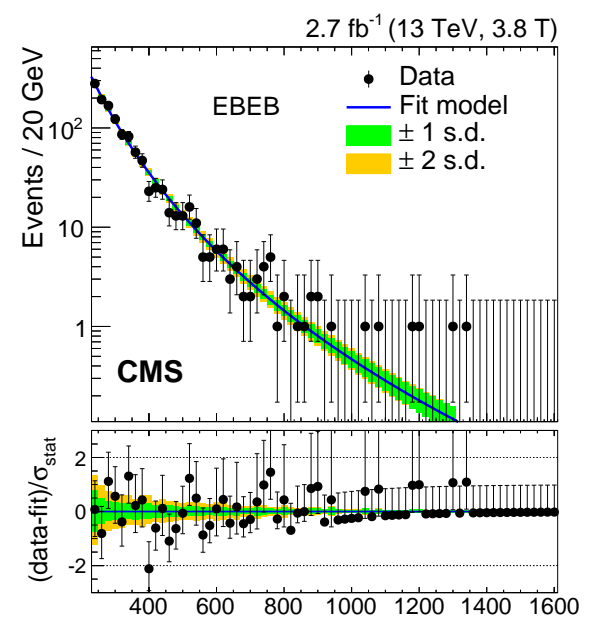

$m_{\gamma \gamma}(\mathrm{GeV})$

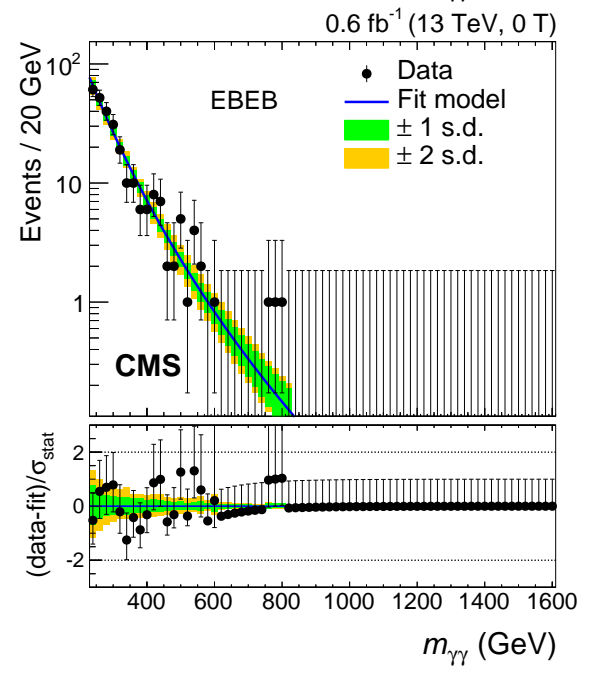

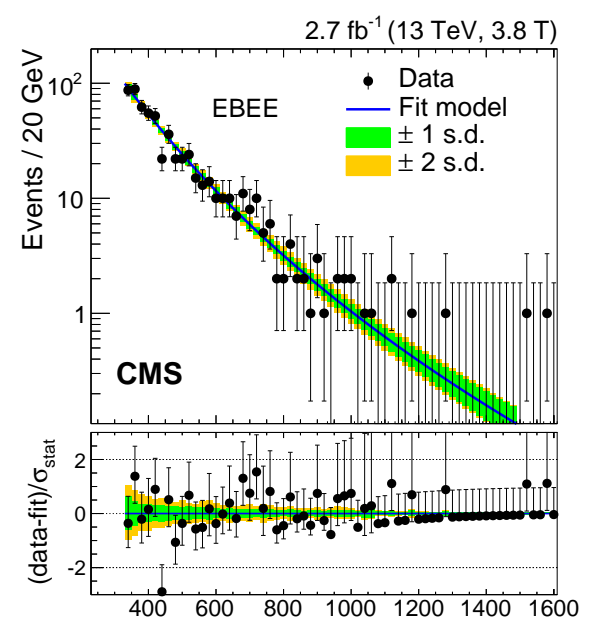

$m_{\gamma \gamma}(\mathrm{GeV})$

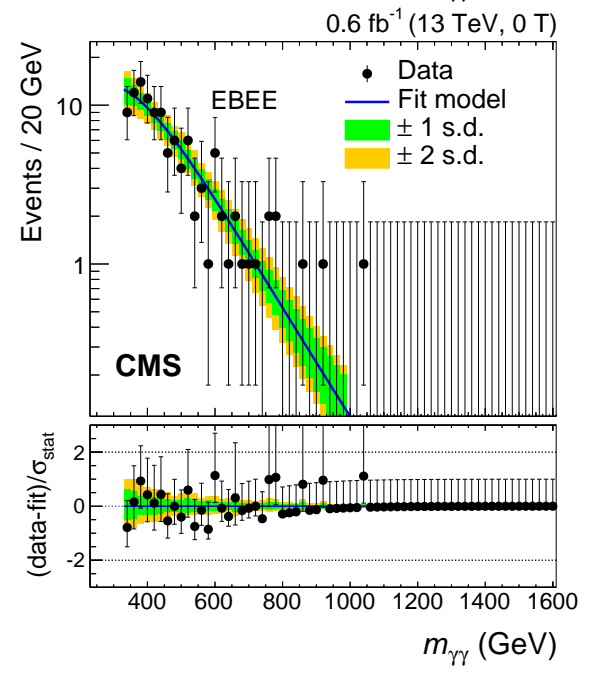

Figure 3: Di-photon invariant mass spectra of the CMS search at $13 \mathrm{TeV}$ [7].

CMS performs a common analysis for spin- 0 and spin-2 resonances, shown in Fig. 3. Events with two photons with $E_{T}>75 \mathrm{GeV}$ are selected. At least one $\gamma$ is required to be reconstructed in the barrel of the electromagnetic calorimeter with $|\eta|<1.44$, where energy resolution is best. Events are categorized according to second photon, that may be either in the barrel or endcap 
$(1.57<|\eta|<2.5)$. In addition events are further categorized according to the state of the CMS magnet, that was switched on in $2.7 / \mathrm{fb}$ of the data and off in $0.6 / \mathrm{fb}$ collected in 2015 . The background is estimated from signal + background fit, where the background is described by a smooth function: $f\left(m_{\gamma \gamma}\right)=m_{\gamma \gamma}^{a+b \times \log \left(m_{\gamma \gamma}\right)}$.
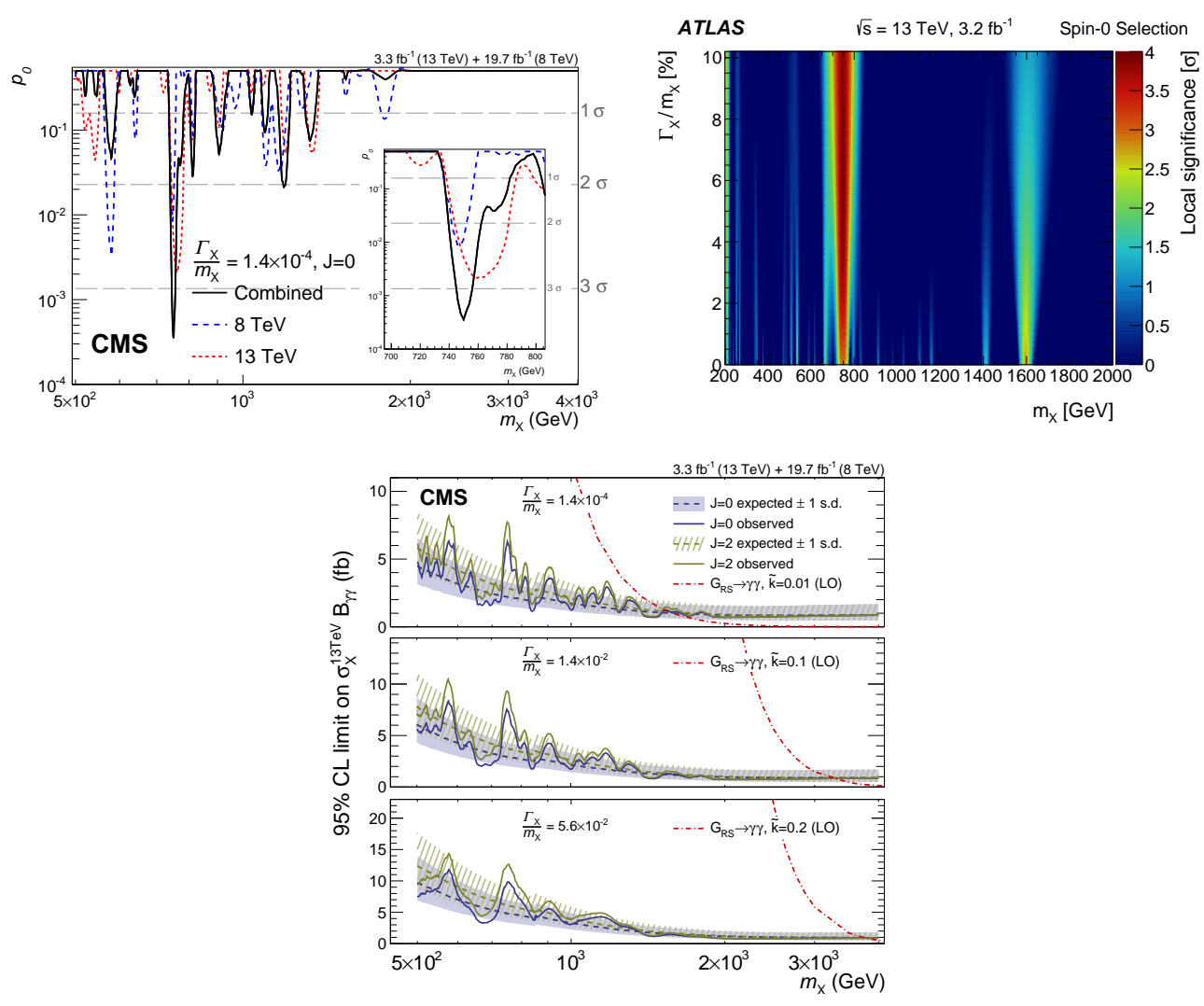

Figure 4: (top left) Combined significance as a function of resonance mass of the $8 \mathrm{TeV}$ and $13 \mathrm{TeV}$ diphoton searches from CMS under the narrow width hypothesis [7]. (top right) Significance as a function of resonance mass and width of the $13 \mathrm{TeV}$ di-photon searches from ATLAS [5]. (bottom) Upper cross section limit on spin- 0 and spin- 2 resonances under three different width hypotheses from the combined 8 and 13 TeV CMS searches [7].

The searches were performed under various resonance width hypotheses from narrower than experimental resolution up to a width of $\Gamma / M \leq 10 \%$ and the resulting limits and significances are shown in Fig. 4. Under a spin-0 hypothesis, ATLAS observes the largest local significance of $3.9 \sigma$ $(2.1 \sigma)$ for a resonance at $750 \mathrm{GeV}$ with $6 \%$ width, and CMS observes the largest local significance of $2.9 \sigma(<1 \sigma)$ at $760 \mathrm{GeV}$ with $1.4 \%$ width. Similar significances are obtained under the spin-2 hypothesis.

ATLAS quantifies the consistency of the 8 and $13 \mathrm{TeV}$ data, and finds values between $1.2 \sigma$ under the hypothesis of a gluon-fusion produced spin- 0 resonance and $3.3 \sigma$ for a qq-annihilation produced spin-2 resonance at $750 \mathrm{GeV}$. CMS performed a statistical combination of the 8 and 13 $\mathrm{TeV}$ data analyses, resulting in an overall local (global) significance of $3.4 \sigma(1.6 \sigma)$ for a spin2 Randall-Sundrum graviton (produced in a mixture of qq-annihilation and gluon-fusion) at 750 $\mathrm{GeV}$. 


\section{4. $\mathrm{Z}+$ photon resonances}

CMS has recently performed a search for spin-0 resonances decaying to $\mathrm{Z} \gamma \rightarrow q q \gamma$, shown in Fig. 5. Events are required to have at least one $\gamma$ with $p_{T}>180 \mathrm{GeV}$ and $p_{T}>0.34 \times M_{Z \gamma}$. In addition one Z-boson candidate is required, that is identified with an $\mathrm{R}=0.8$ jet with $p_{T}>200$ $\mathrm{GeV}, 75<m_{\text {pruned }}<105 \mathrm{GeV}$. Events are organized in two categories according to b-tagging requirements applied to the two subjets of the Z-boson candidate jet, to enhance sensitivity by explicitly selecting $Z \rightarrow b \bar{b}$ events with low background. The background is estimated from a smooth signal+background fit with the background function: $\frac{d N}{M_{Z \gamma}}=P_{0} \times\left(\frac{M_{Z \gamma}}{\sqrt{s}}\right)^{P_{1}+P_{2} \times \log \left(\frac{M_{Z \gamma}}{\sqrt{s}}\right)}$.
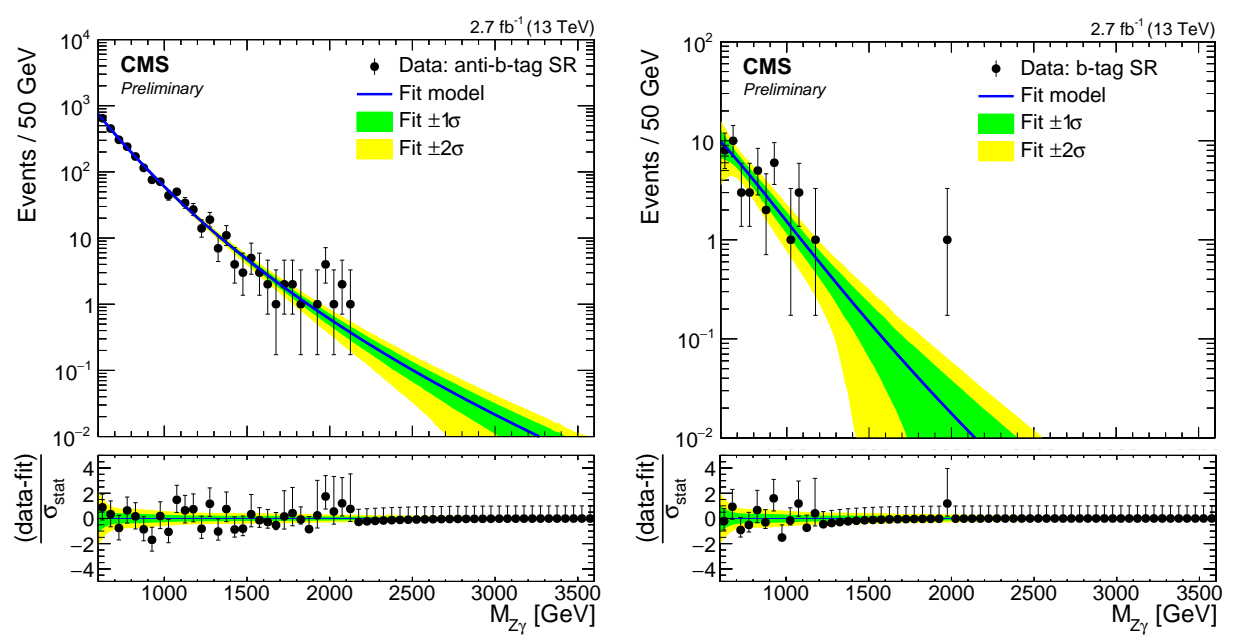

Figure 5: $\mathrm{Z} \gamma \rightarrow q q \gamma$ invariant mass spectra of the CMS search at $13 \mathrm{TeV}$ [13].

The cross section limits from the $\mathrm{Z} \gamma$ resonance searches performed by CMS and ATLAS are summarized in Fig. 6. No significant excess is observed and 95\% C.L. limits for two resonance widths scenarios $(\Gamma / m=0.014 \%, 5.6 \%)$ are set. The searches for $\mathrm{Z} \gamma \rightarrow q q \gamma$ have higher acceptance and thus give best sensitivity at high masses, while the $\mathrm{Z} \gamma \rightarrow l l \gamma$ searches have a lower trigger thresholds and less background, thus give best sensitivity at low masses. CMS has also combined the searches for $\mathrm{Z} \gamma \rightarrow l l \gamma$ at $8 \mathrm{TeV}$ and $13 \mathrm{TeV}$, resulting in a significant gain in sensitivity.

\section{WW/WZ/ZZ resonances}

WW/WZ/ZZ resonances are searched for primarily in final states where at least one W/Z decays to quarks, which are the most sensitive final states at high invariant masses due to the high branching fraction of W/Z to quarks. All possible decay modes of the second W/Z boson are considered as shown in Fig. 7.

The search in the channel where both W/Z bosons decay to quarks from CMS, selects events with two $\mathrm{R}=0.8$ jets with $65<m_{\text {pruned }}<105 \mathrm{GeV}$. Events are organized in three categories depending on if the two pruned jet masses $m_{J 1}$ and $m_{J 2}$ are below or above $85 \mathrm{GeV}$ to reach optimal sensitivity to $\mathrm{WW}, \mathrm{ZZ}$ and $\mathrm{WZ}$ resonances (up to $30 \%$ improvement on cross section limit). Events are further categorized according to $\tau_{2} / \tau_{1}$ into a high purity sample that is most sensitive at low $m_{J J}$ and a high efficiency sample that is most sensitive at high $m_{J J}$. To suppress the large QCD multijet 

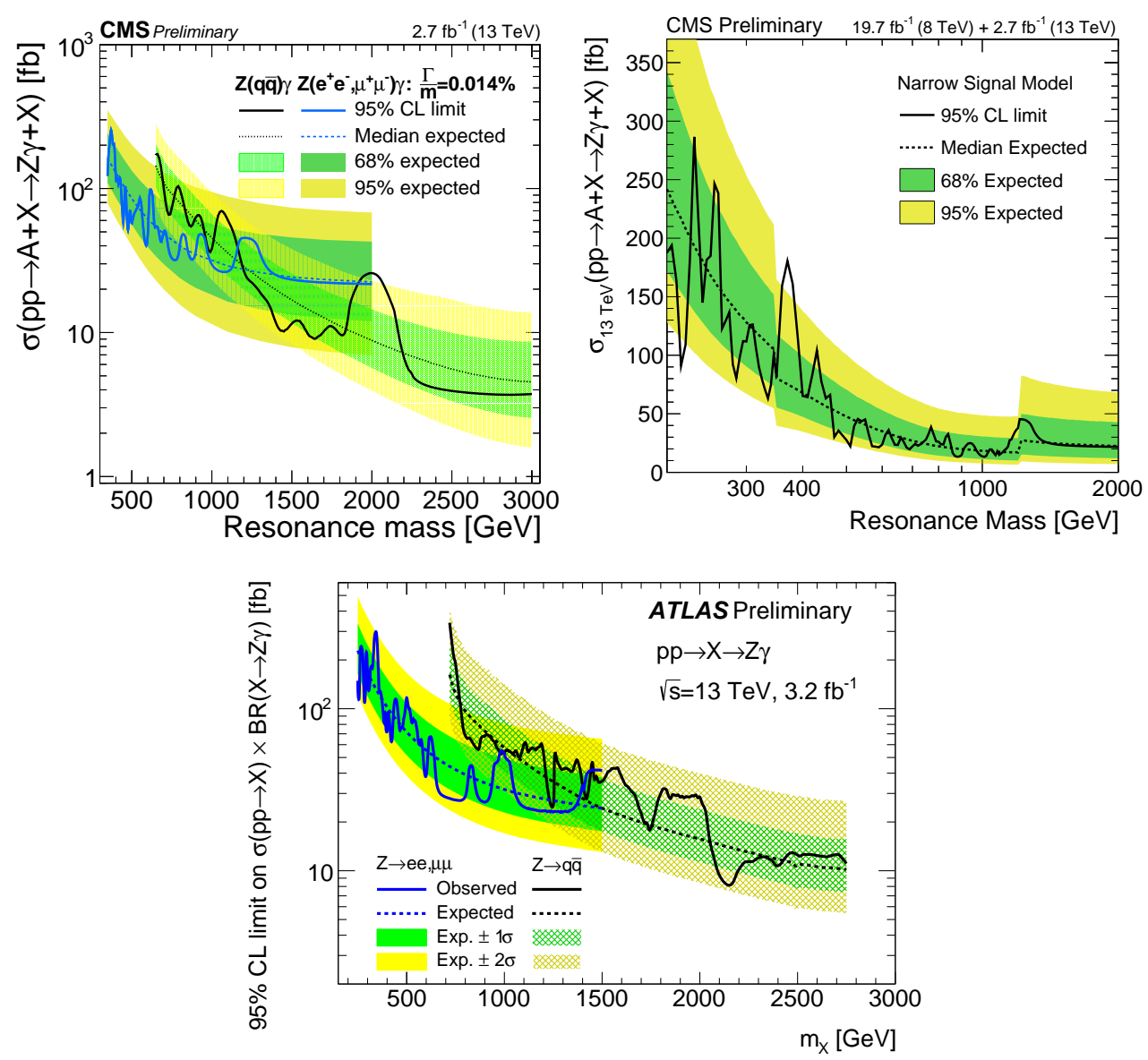

Figure 6: (top left) Cross section upper limits from the CMS $\mathrm{Z} \gamma$ searches at $13 \mathrm{TeV}$ [13]. (top right) Combined cross section upper limits from the $\mathrm{CMS} \mathrm{Z} \gamma \rightarrow l l \gamma$ searches at $8 \mathrm{TeV}$ and $13 \mathrm{TeV}$ [14]. (bottom) Cross section upper limits from the ATLAS Z $\gamma$ searches at $13 \mathrm{TeV}$ [12].

background a requirement on the dijet kinematics $\left|\eta_{J 1}-\eta_{J 2}\right|<1.3$ is applied. The background is estimated from signal + background fit with the function: $\frac{d N}{d m_{j j}}=\frac{P_{0}\left(1-m_{j j} / \sqrt{s}\right)^{P_{1}}}{\left(m_{j j} / \sqrt{s}\right)^{P_{2}}}$. It should be noted that no sign of an excess is observed at $2 \mathrm{TeV}$, where a $3.4 \sigma$ hint if an excess was seen by ATLAS on the 2012 dataset.

In the searches with at least one lepton or neutrino in the final state from ATLAS, the W/Z decaying to quarks is identified with an $\mathrm{R}=1.0$ jet with $p_{T}^{J}>200 \mathrm{GeV}$ and a requirement on $D_{2}^{\beta=1}$ that yields 50\% efficiency. Two separate analyses are performed for final states with $\mathrm{W}$ and $\mathrm{Z}$, requiring $m_{W / Z} \pm 13-15 \mathrm{GeV}$. Three analyses are performed depending on the decay of the second W/Z. The $\mathrm{Z} \rightarrow v v$ analysis selects events with $E_{T}^{\text {miss }}>250 \mathrm{GeV}, \Delta \phi\left(E_{T}^{\text {miss }}\right.$, jets $)>0.6$ and $p_{T}^{\text {miss }}>$ $30 \mathrm{GeV}$. The $\mathrm{W} \rightarrow \mu v / e v$ analysis requires $p_{T}^{\mu / e}>25 \mathrm{GeV}, E_{T}^{m i s s}>100 \mathrm{GeV}, p_{T}^{W}>200 \mathrm{GeV}$ and $>0.4 \times m_{l v J}$, as well as a b-tag-veto against background from $t \bar{t}$ production. The $\mathrm{Z} \rightarrow \mu \mu / e e$ analysis selects events with $p_{T}^{\mu / e}>25 \mathrm{GeV}, 84<m_{e e}<99\left(66<m_{\mu \mu}<116\right), p_{T}^{Z}>0.4 \times m_{l l j}$. The dominant backgrounds from $\mathrm{V}+\mathrm{jets}(t \bar{t})$ are estimated from data in $m_{J}$ (b-tag) sidebands (and $N_{\mu}$ for the $v v$ channel).

CMS has also performed a search specifically optimized for a spin-2 resonance with a mass 

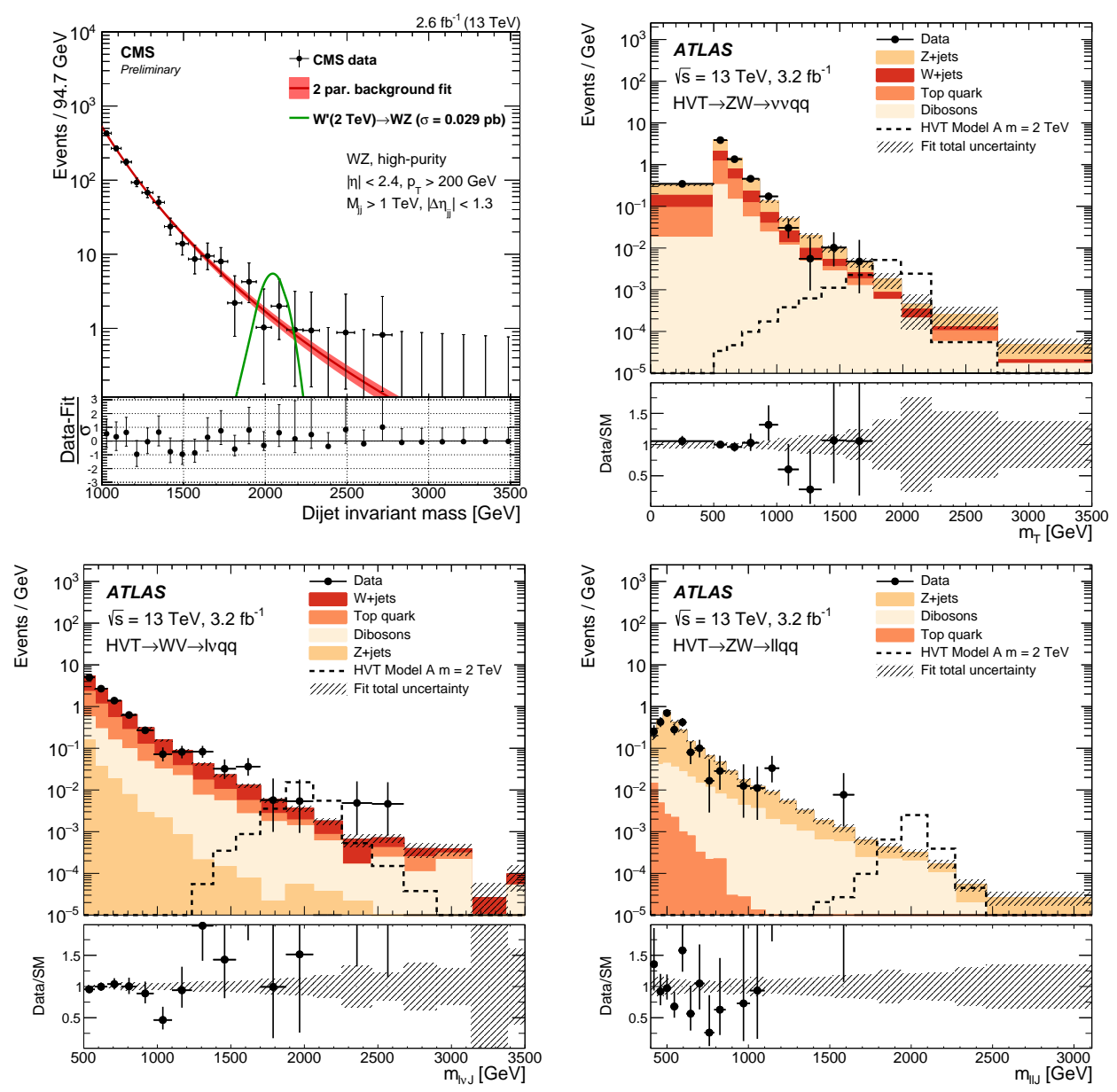

Figure 7: Selected WW/WZ/ZZ invariant mass distributions from the ATLAS [16] and CMS [17] searches at $13 \mathrm{TeV}$.

of $750 \mathrm{GeV}$, whose result is shown in Fig. 8. No excesses has been observed by CMS and ATLAS in the $13 \mathrm{TeV}$ searches. Better mass limits than in the 2012 run of the LHC have been obtained, constraining e.g. the mass of a spin-1 resonance decaying to WZ to $m_{W^{\prime} \rightarrow W Z}>2.3 \mathrm{TeV}$ (HVT model B). A heavy vector triplet model (as predicted e.g. by a composite Higgs model) is also considered, which predicts both spin- $1 W^{\prime \pm}$ and $Z^{\prime}$ (like $\rho^{ \pm}, \rho^{0}$ in nuclear physics). In this model the most stringent mass limit is set at $m_{W^{\prime} / Z^{\prime} \rightarrow W Z / W W}>2.6 \mathrm{TeV}$ (HVT model B). Also scenarios of spin-2 resonances, where a limit of $m_{G *}>1.06 \mathrm{TeV}\left(k / M_{P L}=1.0\right)$ is set, and scenarios of spin-0 resonances, setting a limit of $m_{S}>2.65 \mathrm{TeV}$ (unsuppressed scenario), have been evaluated.

\section{6. $\mathrm{WH} / \mathrm{ZH}$ resonances}

$\mathrm{WH} / \mathrm{ZH}$ resonances at high invariant masses are searched for primarily in final states where the Higgs decays to b-quarks due to the high branching fraction. In the CMS searches at $13 \mathrm{TeV}$, the Higgs boson is identified with a $\mathrm{R}=0.8$ jet with $p_{T}^{J}>200 \mathrm{GeV}$ and $105<m_{\text {pruned }}<135$, shown in Fig. 9. Events are categorized according to the number of b-tagged subjets (1 or 2 ) of the Higgs candidate jet, where the $2 \mathrm{~b}$-tag category is most sensitive at low masses due to the good 

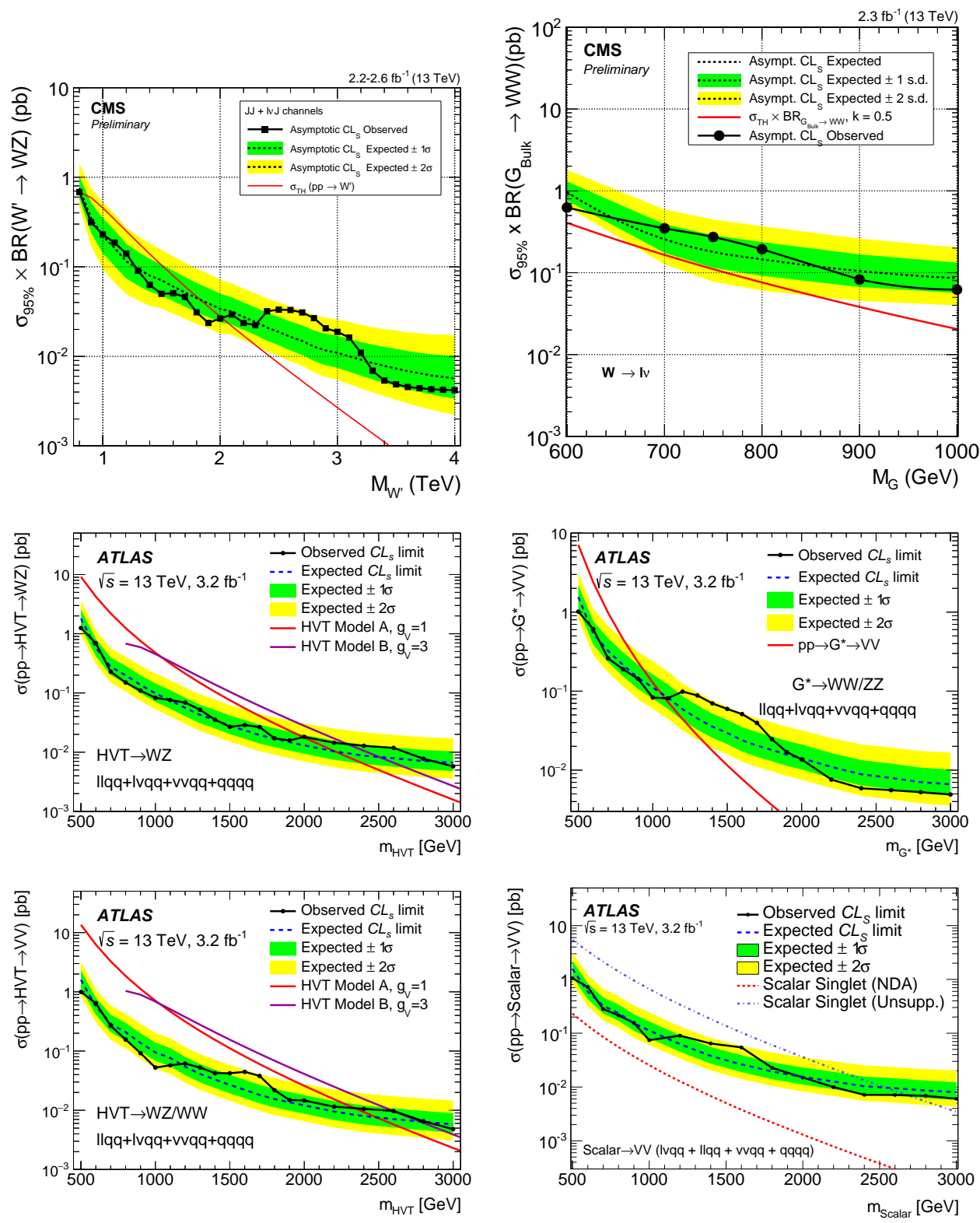

Figure 8: (top left) Cross section limit on spin-1 WZ resonances from the $13 \mathrm{TeV}$ CMS search [17]. (top right) Cross section limit on spin-2 WW resonances from the $13 \mathrm{TeV}$ CMS optimized for a mass of 750 $\mathrm{GeV}$ [18]. (middle left) Cross section limit on spin-1 WZ resonances from the $13 \mathrm{TeV}$ ATLAS search [16]. (middle right) Cross section limit on spin-2 WW/ZZ resonances from the $13 \mathrm{TeV}$ ATLAS search [16]. (bottom left) Cross section limit on spin-1 WZ/WW triplet resonances from the 13 TeV ATLAS search [16]. (bottom right) Cross section limit on spin-0 WW/ZZ resonances from the $13 \mathrm{TeV}$ ATLAS search [16].

background rejection, while the $1 \mathrm{~b}$-tag category recovers efficiency for optimal sensitivity at high masses. Otherwise the analysis strategy is very similar to the corresponding WW/WZ/ZZ analyses. The $v v$ analysis requires $E_{T}^{\text {miss }}>200 \mathrm{GeV}, \Delta \phi\left(E_{T}^{\text {miss }}, J\right)>2$ and a b-tag-veto. The $\mu v / \mathrm{e} v$ analysis requires $p_{T}^{\mu}>55 \mathrm{GeV}\left(p_{T}^{e}>135, E_{T}^{\text {miss }}>80 \mathrm{GeV}\right), p_{T}^{W}>200 \mathrm{GeV}$ and a b-tag-veto. The $\mu \mu /$ ee analysis requires $p_{T}^{\mu}>55\left(p_{T}^{e}>135\right) \mathrm{GeV}, 70<m_{l l}<110, p_{T}^{Z}>200 \mathrm{GeV}, \Delta \phi(l l, J)>2.5$. The 
main backgrounds from $\mathrm{V}+\mathrm{jets}(t \bar{t})$ are estimated from data in $m_{J}$ (b-tag-veto) sidebands.
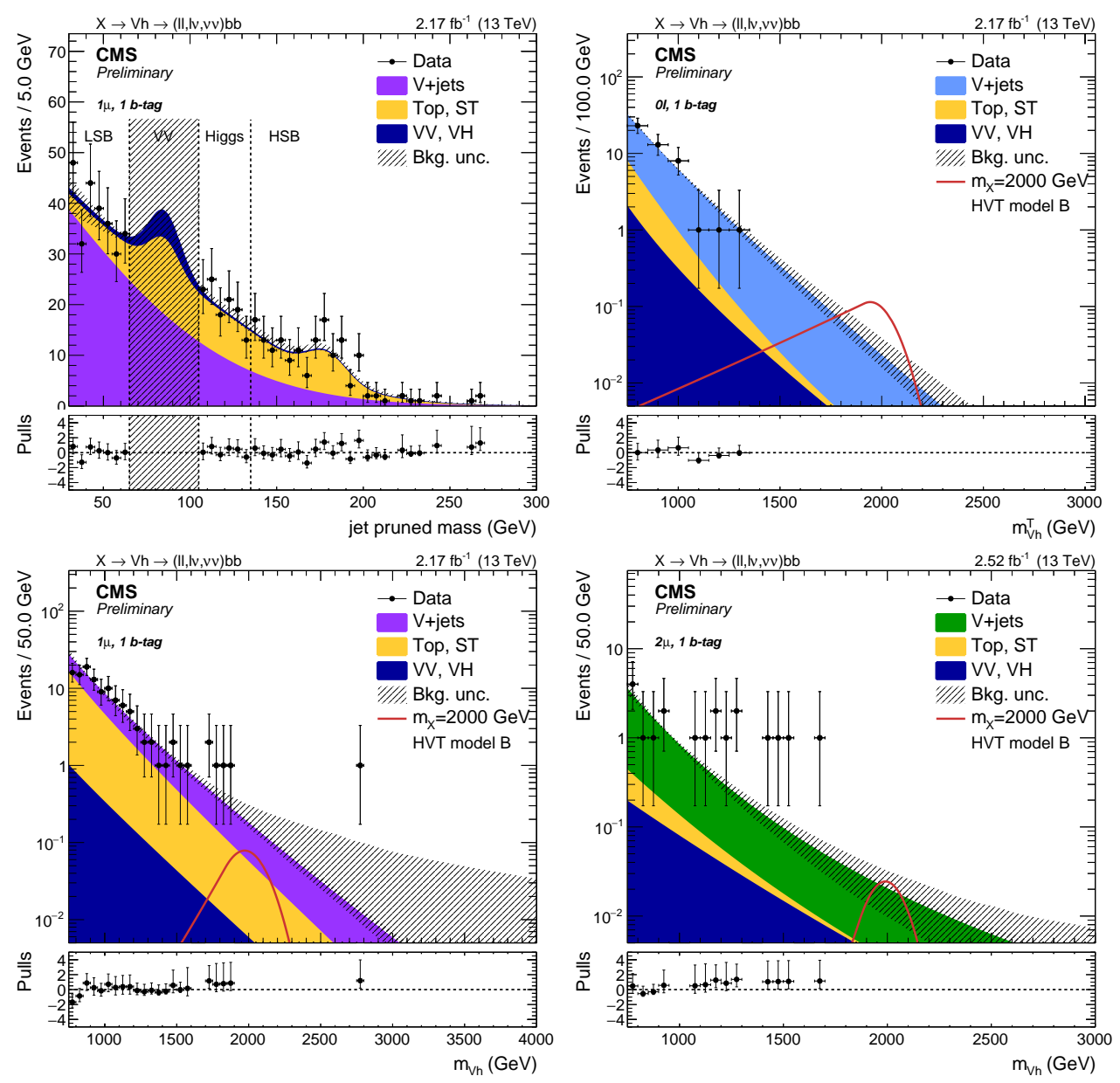

Figure 9: (top left) Pruned jet mass distribution used in the background estimation procedure of the WH/ZH CMS searches at $13 \mathrm{TeV}$ [21]. (other plots) Selected WH/ZH invariant mass distributions from the CMS searches at $13 \mathrm{TeV}$ [21].

No significant excess is observed over background as shown in Fig. 10. Spin-1 resonances with masses $m_{W^{\prime}}<2.2 \mathrm{TeV}$ and $m_{Z^{\prime}}<1.7 \mathrm{TeV}$ are excluded in HVT model B.

\section{WW/WZ/ZZ/WH/ZH combination}

Since the various di-boson search channels and also the two datasets at 8 and $13 \mathrm{TeV}$ presented above have very similar sensitivity to the new physics scenarios of interest, CMS has performed a statistical combination of WW/WZ/ZZ/WH/ZH searches. W', Z', heavy vector triplet (W' $\left.+Z^{\prime}\right)$ and bulk graviton interpretations are provided. Fig. 11 (top left) compares the sensitivity of various di-boson search channels on the $8 \mathrm{TeV}$ dataset, where the combination can improve sensitivity over the most sensitive channel by a factor 2. Fig. 11 (top right) and (bottom left) compare the sensitivity of various di-boson search channels on the 8 and $13 \mathrm{TeV}$ dataset. For a spin-1 resonance produced via qq-annihilation, $13 \mathrm{TeV}$ searches are better than $8 \mathrm{TeV}$ searches above $\sim 1.5 \mathrm{TeV}$, while for 

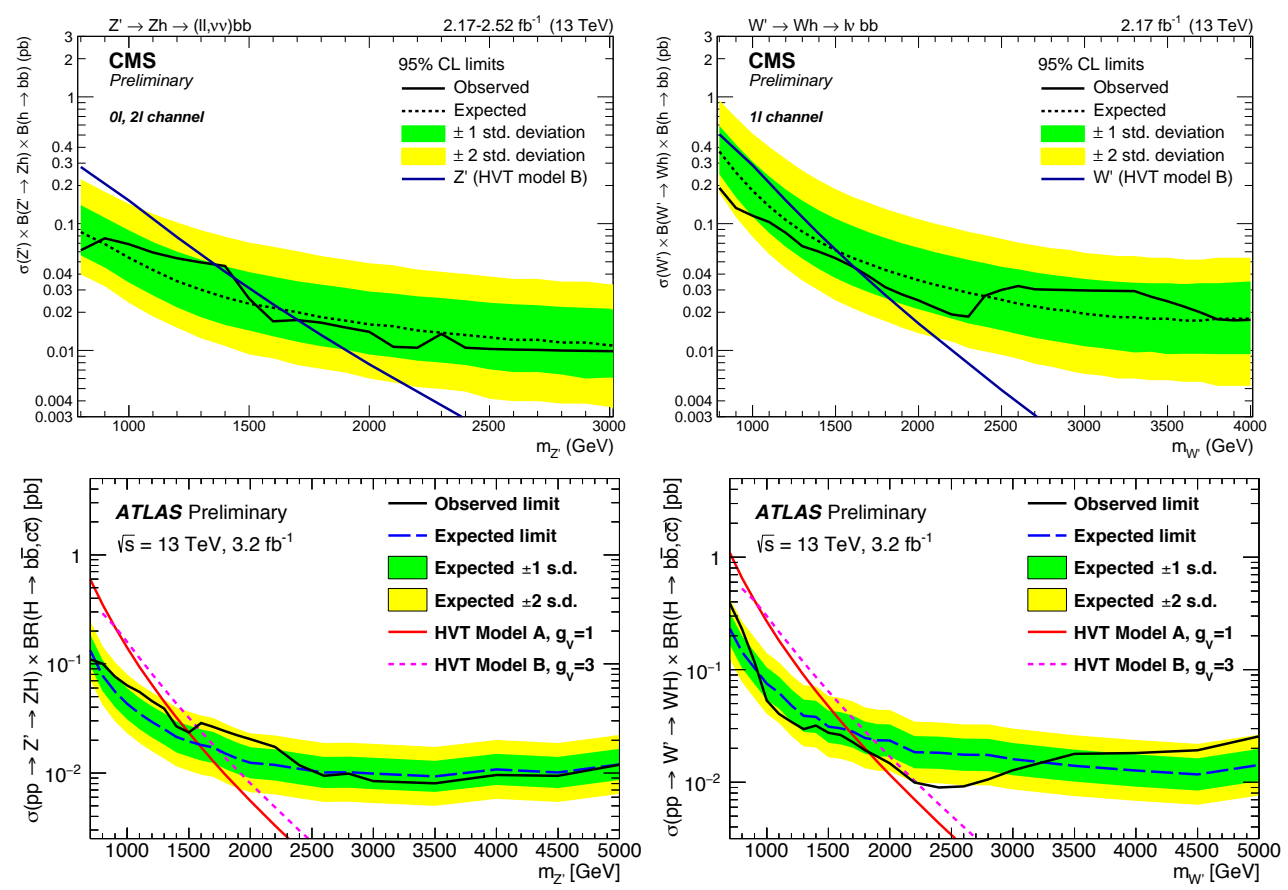

Figure 10: Cross section limit on spin-1 (left) $\mathrm{WH}$ and (right) $\mathrm{ZH}$ resonances from the $13 \mathrm{TeV}$ (top) CMS [21] and (bottom) ATLAS [20] searches.

a spin-2 resonance produced via gluon-fusion, $13 \mathrm{TeV}$ searches are better than $8 \mathrm{TeV}$ searches already above $\sim 0.5 \mathrm{TeV}$. Fig. 11 (bottom right) shows the limit on the coupling to fermions and coupling to bosons for a spin-1 vector triplet resonance from the combination of 8 and $13 \mathrm{TeV}$ CMS searches.

The most significant excess from the 2012 dataset in CMS had a local significance of $2.2 \sigma$ for a $\mathrm{W}^{\prime} \rightarrow \mathrm{WH}$ at $1.8 \mathrm{TeV}$. Combining all $8 \mathrm{TeV} \mathrm{VV}+\mathrm{VH}$ searches it remains $2.2 \sigma$ in the W' hypothesis. Combining all $8+13 \mathrm{TeV} \mathrm{VV}+\mathrm{VH}$ searches, it is reduced to $0.9 \sigma$ in the $\mathrm{W}^{\prime}$ hypothesis, therefore not supporting the existence of a resonance around $2 \mathrm{TeV}$.

\section{HH resonances}

$\mathrm{HH}$ resonances at high invariant masses are searched for primarily in final states where the Higgs bosons decay to b-quarks due to the high branching fraction. In the ATLAS searches at $13 \mathrm{TeV}$, spin 0 and 2 resonance decaying to $\mathrm{HH} \rightarrow$ bbbb are searched for in two event topologies adapted to the boost of the Higgs boson, shown in Fig. 12. In the resolved analysis (low boost), 4 btagged jets with $m_{j j} \sim m_{H}$ are selected, while in the boosted analysis, two $\mathrm{R}=1.0$ jets with $m_{J} \sim m_{H}$, which have 3-4 b-tagged matched trackjets associated, are required. The main backgrounds from QCD multijets and $t \bar{t}$ production are estimated from data in $N_{b-t a g s}, m_{J 1}$ and $m_{J 2}$ sidebands.

An upper limit on non-resonant $\mathrm{HH}$ production is set at $\sigma(p p \rightarrow h h \rightarrow b b b b)<1.22 \mathrm{pb}$, that can be compared to the corresponding standard model production cross section of $\sigma(p p \rightarrow h h \rightarrow$ $b b b b)=12.9_{-1.6}^{+1.5} \mathrm{fb}$. 

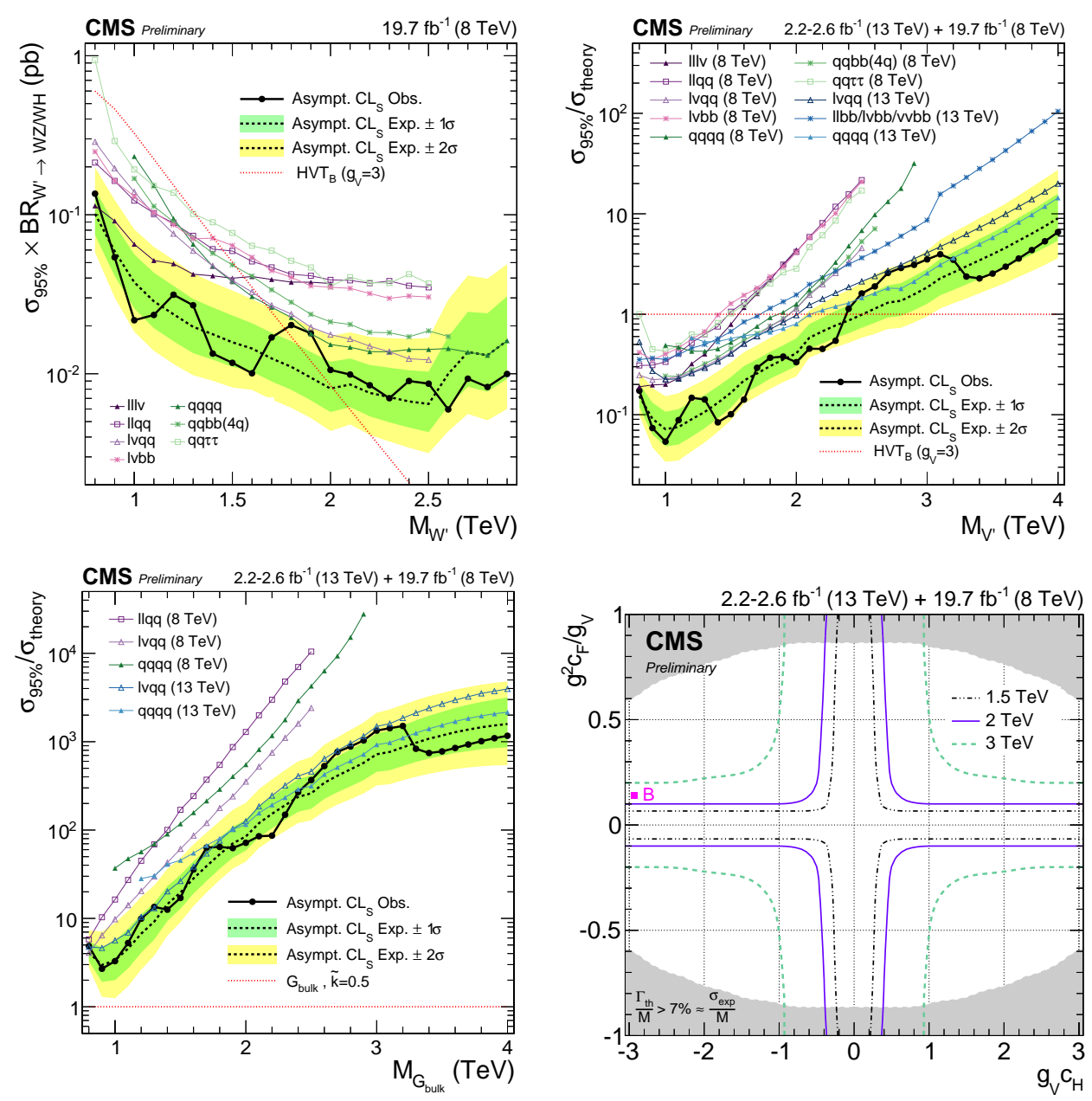

Figure 11: (top left) Cross section limit on spin-1 WZ resonances from the combination of 8 TeV CMS searches [19]. (top right) Cross section limit on spin-1 WZ resonances from the combination of 8 and $13 \mathrm{TeV}$ CMS searches [19]. (bottom left) Cross section limit on spin-2 WW/ZZ resonances from the combination of 8 and 13 TeV CMS searches [19]. (bottom right) Limit on the coupling to fermions and coupling to bosons for a spin-1 vector triplet resonance from the combination of 8 and $13 \mathrm{TeV}$ CMS searches [19].

\section{Low mass boson resonances}

Theories with hidden-sector particles that may resolve our current lack of evidence for a dark matter particle candidate, predict new low mass bosons. LHCb has searched for such low mass bosons $\chi \rightarrow \mu \mu$ with coupling to the top quark, with the production mechanism demonstrated in Fig. 13 (top). The search is sensitive to the $\chi$ decay time by reconstructing the $K^{* 0} \rightarrow K^{+} \pi^{-}$ decay vertex. Background rejection is enhanced with an MVA-based selection and the resulting $\chi \rightarrow \mu \mu$ candidate mass spectrum is shown in Fig. 13 (bottom). The background is estimated from neighboring 4-6 bins in the mass spectrum. This search puts the most stringent constraints to date on the production cross section of such resonances covering masses in the range 200-4400 MeV. 

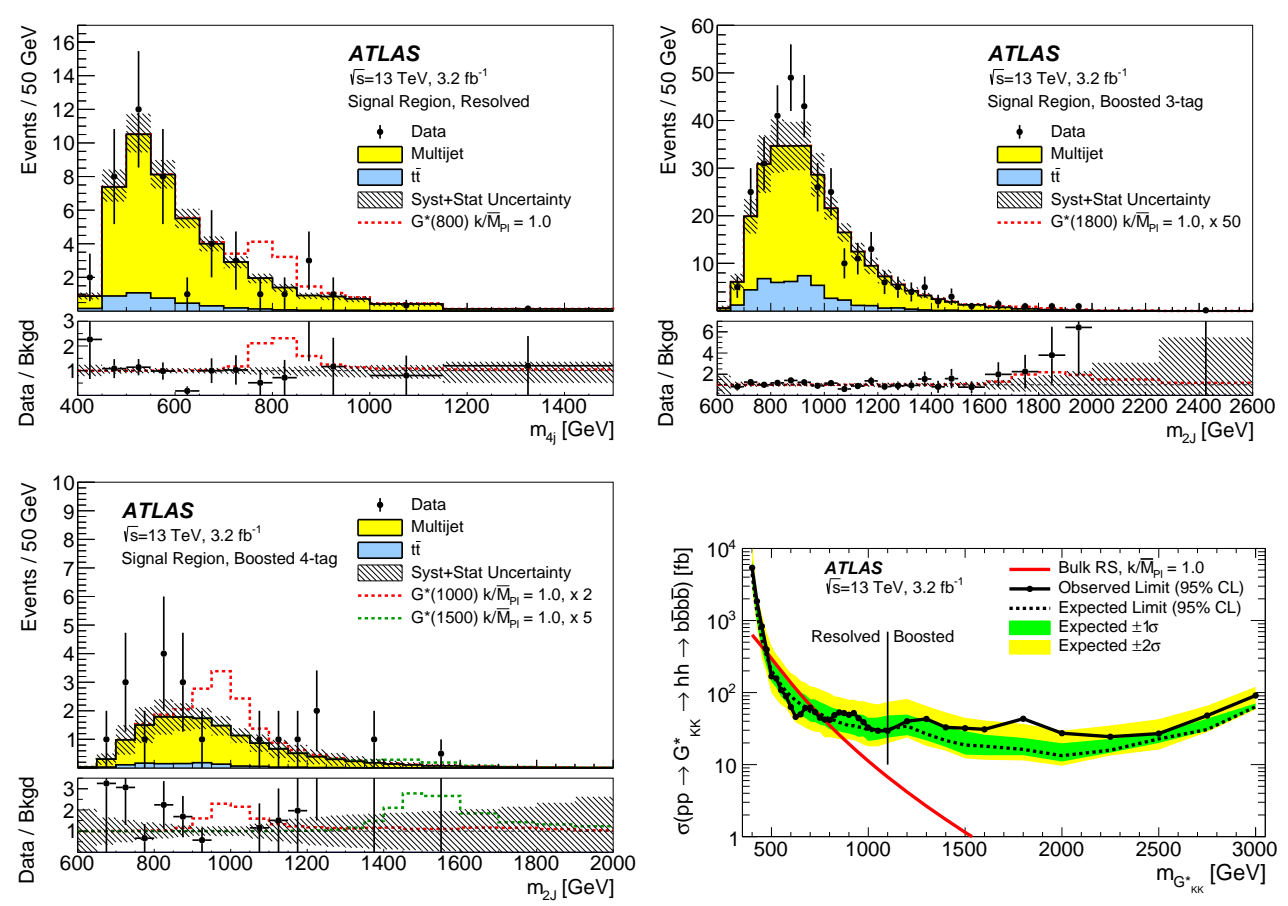

Figure 12: (top) and (bottom left) Selected HH invariant mass distributions from the ATLAS searches at 13 $\mathrm{TeV}$ [22]. (bottom right) Cross section limit on spin-2 HH resonances from the $13 \mathrm{TeV}$ ATLAS search [22].

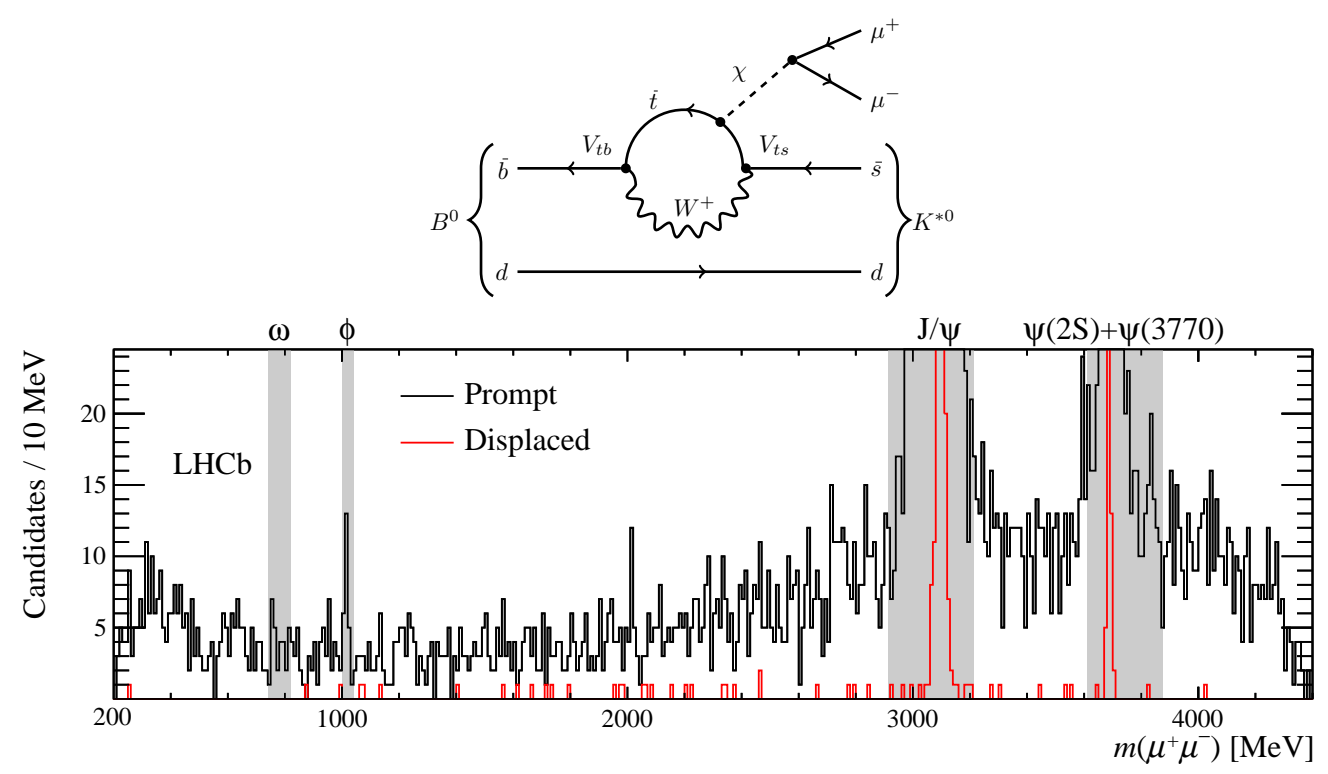

Figure 13: (top) Feynman diagram for the low mass boson production searched for by $\mathrm{LHCb}$ with the 8 $\mathrm{TeV}$ data [23]. (bottom) Invariant mass spectrum of $\chi \rightarrow \mu \mu$ candidates [23].

\section{Conclusions}

Combining the searches in 8 and $13 \mathrm{TeV}$ data, one can conclude a solid "maybe" on the hint of a resonance at $750 \mathrm{GeV}$ in the 2015 dataset. Shortly after the LHCP conference, the 2016 dataset 
of the LHC gives the final answer to this question. CMS and ATLAS explore di-boson resonance with $>\mathrm{TeV}$ masses in all important final states. Interpretations cover spin-0, spin-1 (HVT), spin-2 (RSG) scenarios. The analyses with $13 \mathrm{TeV}$ data already supersede the $8 \mathrm{TeV}$ searches at $>\mathrm{TeV}$ masses and set the most stringent mass limits on $\mathrm{W}^{\prime} / \mathrm{Z}^{\prime} / G^{*}$ resonances. The combination of $8+13$ $\mathrm{TeV}$ VV+VH searches disfavors the hint of a bump at $2 \mathrm{TeV}$ seen in the 2012 dataset.

\section{References}

[1] ATLAS Collaboration, The ATLAS experiment at the CERN Large Hadron Collider, JINST 0803:S08003, 2008

[2] CMS Collaboration, The CMS experiment at the CERN LHC, JINST 0803:S08004, 2008

[3] LHCb Collaboration, The LHCb Detector at the LHC, JINST 0803:S08005, 2008

[4] ATLAS Collaboration, Search for resonances decaying to photon pairs in 3.2 /fb of pp collisions at $\sqrt{s}=13$ TeV with the ATLAS detector, ATLAS-CONF-2015-081 (2015) http://cds.cern.ch/record/2114853

[5] ATLAS Collaboration, Search for resonances in diphoton events at $\sqrt{s}=13$ TeV with the ATLAS detector, JHEP 09 (2016) 001

[6] CMS Collaboration, Search for new physics in high mass diphoton events in proton-proton collisions at $\sqrt{s}=13 \mathrm{TeV}$, CMS-PAS-EXO-15-004 (2015) http://cds.cern.ch/record/2114808

[7] CMS Collaboration, Search for resonant production of high-mass photon pairs in proton-proton collisions at $\sqrt{s}=8$ and $13 \mathrm{TeV}$, Phys. Rev. Lett. 117 (2016) 051802

[8] ATLAS Collaboration, Search for Scalar Diphoton Resonances in the Mass Range 65-600 GeV with the ATLAS Detector in pp Collision Data at $\sqrt{s}=8$ TeV, Phys. Rev. Lett. 113 (2014) 171801

[9] ATLAS Collaboration, Search for high-mass diphoton resonances in pp collisions at $\sqrt{s}=8 \mathrm{TeV}$ with the ATLAS detector, Phys. Rev. D 92, 032004 (2015)

[10] CMS Collaboration, Search for High-Mass Diphoton Resonances in pp Collisions at $\sqrt{s}=8$ TeV with the CMS Detector, CMS-PAS-EXO-12-045 (2015) http://cds.cern.ch/record/2017806

[11] CMS Collaboration, Search for diphoton resonances in the mass range from 150 to $850 \mathrm{GeV}$ in pp collisions at $\sqrt{s}=8 \mathrm{TeV}$, Phys. Lett. B 750 (2015) 494

[12] ATLAS Collaboration, Search for heavy resonances decaying to a $Z$ boson and a photon in pp collisions at $\sqrt{s}=13 \mathrm{TeV}$ with the ATLAS detector, ATLAS-CONF-2016-010 (2016) http://cds.cern.ch/record/2139795

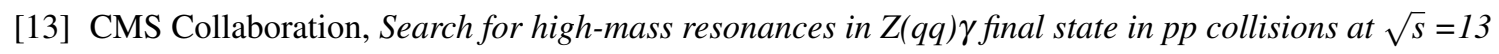
TeV with 2.7 /fb, CMS-PAS-EXO-16-020 (2016) http://cds.cern.ch/record/2161129

[14] CMS Collaboration, Combination of searches for high-mass resonances in Zgamma final states in proton-proton collisions at sqrts 8 and $13 \mathrm{TeV}$, CMS-PAS-EXO-16-021 (2016) http://cds.cern.ch/record/2160238

[15] ATLAS Collaboration, Search for new resonances in W $\gamma$ and $Z \gamma$ Final States in pp Collisions at $\sqrt{s}=8$ TeV with the ATLAS Detector, Phys.Lett. B738 (2014) 428-447

[16] ATLAS Collaboration, Searches for heavy diboson resonances in pp collisions at $\sqrt{s}=13 \mathrm{TeV}$ with the ATLAS detector, submitted to JHEP (2016) https://arxiv.org/abs/1606.04833 
[17] CMS Collaboration, Search for massive resonances decaying into pairs of boosted $W$ and $Z$ bosons at $\sqrt{s}=13 \mathrm{TeV}$, CMS-PAS-EXO-15-002 (2016) http://cds.cern.ch/record/2117062

[18] CMS Collaboration, Search for WW in semileptonic final states: low mass extension, CMS-PAS-B2G-16-004 (2016) http://cds.cern.ch/record/2141101

[19] CMS Collaboration, Combination of searches for $W W, W Z, Z Z, W H$, and $Z H$ resonances at $\sqrt{s}=8$ and $13 \mathrm{TeV}$, CMS-PAS-B2G-16-007 (2016) http://cds.cern.ch/record/2154306

[20] ATLAS Collaboration, Search for new resonances decaying to a $W$ or $Z$ boson and a Higgs boson in the llbb, lvbb, and vvbb channels with pp collisions at $\sqrt{s}=13 \mathrm{TeV}$ with the ATLAS detector, Submitted to PLB (2016) http://arxiv.org/abs/1607.05621

[21] CMS Collaboration, Search for heavy resonances decaying into a vector boson and a Higgs boson in the (ll, lnu, nunu) bb final state, CMS-PAS-B2G-16-003 (2016) http://cds.cern.ch/record/2141576

[22] ATLAS Collaboration, Search for pair production of Higgs bosons in the bbøbbø final state using proton-proton collisions at $\sqrt{s}=13$ TeV with the ATLAS detector, Phys. Rev. D 94, 052002 (2016)

[23] LHCb Collaboration, Search for Hidden-Sector Bosons in $B^{0} \rightarrow K^{* 0} \mu^{+} \mu^{-}$Decays, Phys. Rev. Lett. 115 (2015) 161802

[24] ATLAS Collaboration, Identification of boosted, hadronically decaying $W$ bosons and comparisons with ATLAS data taken at $\sqrt{s}=8 \mathrm{TeV}$, Eur. Phys. J. C 76(3) (2016) 1-47

[25] CMS Collaboration, Identification techniques for highly boosted W bosons that decay into hadrons, JHEP 12 (2014) 017

[26] ATLAS Collaboration, Expected Performance of Boosted Higgs $(\rightarrow b \bar{b})$ Boson Identification with the ATLAS Detector at $\sqrt{s}=13 \mathrm{TeV}$, ATL-PHYS-PUB-2015-035 (2015) http://cds.cern.ch/record/2042155

[27] CMS Collaboration, Identification of b quark jets at the CMS Experiment in the LHC Run 2, CMS-PAS-BTV-15-001 (2016) http://cds.cern.ch/record/2138504 